\title{
Successive Nonstatistical and Statistical Approaches for the Improved Antibiotic Activity of Rare Actinomycete Nonomuraea sp. JAJ18
}

\author{
Polpass Arul Jose and Solomon Robinson David Jebakumar \\ Department of Molecular Microbiology, School of Biotechnology, Madurai Kamaraj University, Madurai 625 021, India \\ Correspondence should be addressed to Solomon Robinson David Jebakumar; jsolomon_mrna@yahoo.com
}

Received 3 February 2014; Accepted 7 August 2014; Published 3 September 2014

Academic Editor: Paul M. Tulkens

Copyright (C) 2014 P. Arul Jose and S. R. D. Jebakumar. This is an open access article distributed under the Creative Commons Attribution License, which permits unrestricted use, distribution, and reproduction in any medium, provided the original work is properly cited.

\begin{abstract}
The selection and optimization of nutritional constituents as well as their levels for the improved production of antibiotic by Nonomuraea sp. JAJ18 were carried out using combination of both nonstatistical one-factor-at-a-time (OFAT) method and statistical response surface methodology (RSM). Using OFAT method, starch and $\left(\mathrm{NH}_{4}\right)_{2} \mathrm{SO}_{4}$ were identified as suitable carbon and nitrogen sources, respectively. Subsequently, starch, $\mathrm{NaCl}$, and $\mathrm{MgSO}_{4} \cdot 7 \mathrm{H}_{2} \mathrm{O}$ were recognized as the most significant media components with confidence level of above 95\% using the Plackett-Burman design. The levels of the three media components were further optimized using RSM employed with Box-Behnken design. Accordingly, a second-order polynomial regression model was fitted into the experimental data. By analyzing the response surface plots as well as using numerical optimization method, the optimal levels for starch, $\mathrm{NaCl}$, and $\mathrm{MgSO}_{4} \cdot 7 \mathrm{H}_{2} \mathrm{O}$ were determined as $15.6 \mathrm{~g} / \mathrm{L}, 0.8 \mathrm{~g} / \mathrm{L}$, and $1.98 \mathrm{~g} / \mathrm{L}$, respectively. With the optimized medium, $15.5 \%$ increase was observed in antibiotic activity of JAJ18. Results further support the use of RSM for media optimization. To the best of our knowledge, this is the first report of statistical media optimization for antibiotic production in rare actinomycete Nonomuraea species, which will be useful for the development of Nonomuraea cultivation process for efficient antibiotic production on a large scale.
\end{abstract}

\section{Introduction}

Since the report of streptomycin, actinomycetes, especially streptomycetes, have been shown to be a prime source of antibiotics. However, the likelihood of finding novel compounds has dwindled due to extensive studies on the ubiquitous species [1]. In the recent years, rare actinomycetes are considered as potential store house of novel antibiotics [2-4] and the current antibiotic screening programs have been oriented towards rare actinomycetes derived from unexplored environments $[5,6]$. However, studies on development of ideal production medium and culture conditions for consistent production of antibiotics from these rare actinomycetes remain scarce with limited reports.

Improving the antibiotic production is prerequisite to achieve adequate antibiotic yield for evaluating its potential and novelty. Antibiotic producing ability of the actinomycetes is not a static property and it is highly influenced by culture conditions and media components [7-9]. Therefore, designing an appropriate fermentation medium and conditions has crucial importance in improving the antibiotic yield and easing the cost of production [10,11]. Designing an appropriate cultivation system for producer strains require a series of trials like selection of basal medium, selection of carbon and nitrogen sources, optimization of the physical parameters, screening for medium components which have significant influence on production of particular compound, and optimization of influencing medium components [12]. Selection of carbon and nitrogen sources can be done using a classical method, one-factor-at-a-time (OFAT) approach by changing one independent variable while fixing all the others at a fixed level $[12,13]$. Subsequently, screening of medium components for their significant effect on product formation can be done using statistical factorial designs like PlackettBurman design (PBD). The Plackett-Burman design is a wellestablished and widely used statistical design technique for 
the screening of the medium components in shake flask [11, 14]. The significant media components can then be optimized using response surface methodology (RSM) [12, 15].

The aim of the present work was to design and optimize cultural parameters as well as significant media components to achieve improved antibiotic production in rare actinomycete, Nonomuraea sp. JAJ18, which was previously isolated from an Indian coastal solar saltern [16]. The study was executed using a successive optimization strategy, which includes, selection of basal medium, selection of carbon and nitrogen sources using OFAT, selection of media components that significantly influence the antibiotic production using $\mathrm{PBD}$, and optimization of these media components using RSM with Box-Behnken design.

\section{Materials and Methods}

2.1. Strain JAJ18. The rare actinomycete strain JAJ18 used in this study was previously isolated from a coastal solar saltern established at Tuticorin, India [16]. It was identified and designated as Nonomuraea sp. JAJ18 and the pure culture was maintained at $4^{\circ} \mathrm{C}$ on modified inorganic salt agar slants which contained $10 \mathrm{~g}$ of starch, $4.0 \mathrm{~g}$ of yeast extract, $20 \mathrm{~g}$ of $\mathrm{NaCl}, 2.0 \mathrm{~g}$ of $\left(\mathrm{NH}_{4}\right)_{2} \mathrm{SO}_{4}, 1.0 \mathrm{~g} \mathrm{MgSO} \cdot 7 \mathrm{H}_{2} \mathrm{O}, 1.0 \mathrm{~g}$ $\mathrm{K}_{2} \mathrm{HPO}_{4}$, and $22 \mathrm{~g}$ of agar in $1.0 \mathrm{~L}$ of distilled water. The bacterial indicator strain, Bacillus subtilis MTCC 441 used for antibiotic assay was maintained over Mueller Hinton agar slants. The MTCC culture was obtained from Microbial Type Culture Collection, IMTECH, India.

2.2. Extraction of Antibiotic Substance. Fermentation broth of JAJ18 was centrifuged at $10000 \mathrm{rpm}$ for $10 \mathrm{~min}$ and the cellfree supernatant was recovered. Ethyl acetate was added to the supernatant in 1:1 proportion and the mixture was agitated for $20 \mathrm{~min}$. The solvent layer containing antibiotic substance was separated from broth and it was further centrifuged at $5000 \mathrm{rpm}$ for $15 \mathrm{~min}$ to remove traces of fermentation broth. The crude extract was concentrated tenfold using a rotational vacuum concentrator and used for antibiotic assay.

2.3. Antibiotic Assay. Antibiotic assays were carried out in triplicates against Bacillus subtilis MTCC 441 by agar diffusion plate assay $[12,17]$. Sterile discs of $6 \mathrm{~mm}$ in diameter were impregnated with crude extract, dried, and placed on nutrient agar plate inoculated with $B$. subtilis suspension adjusted to a McFarland standard of 0.5 , which is equivalent to $1.5 \times 10^{8} \mathrm{CFU} / \mathrm{mL}$. A sterile disc impregnated with ethyl acetate was used as control. The plates were incubated at $37^{\circ} \mathrm{C}$ for $24 \mathrm{~h}$ and the inhibition zone formed around the disc was measured in millimetre. Maxwell et al. [17] and Wang et al. [12] confirmed that the size of the zones of inhibition can be considered as a measure of antibiotic titre. Therefore, the antibiotic activity was expressed as units of activity per millilitre crude substance of the culture, where $1 \mathrm{U}$ was defined as a $1.0 \mathrm{~mm}$ annular clearing around the antibiotic disk.
2.4. Determination of Growth. The mycelial pellet was collected by centrifugation at $10000 \mathrm{rpm}$ for $10 \mathrm{~min}$ and dried at $60^{\circ} \mathrm{C}$. The dried pellet was weighed to determine the growth. The growth in terms of mycelial biomass accumulation was expressed as $\mathrm{g} / \mathrm{L}$ of the culture medium.

2.5. Experimental Overview of Media Optimization. In preliminary experiments, various culture conditions, carbon sources, and nitrogen sources were evaluated for their suitability to sustain good antibiotic activity by strain JAJ18. An appropriate basal medium with chosen carbon and nitrogen sources was taken for statistical screening and further optimization using PBD and RSM, respectively.

2.6. Seed Culture. Spore suspension of JAJ18 was prepared in Milli-Q water from the culture grown on ISP4 medium supplemented with $4 \%$ of yeast extract and $0.5 \%$ of $\mathrm{NaCl}$ $(\mathrm{w} / \mathrm{v})$ at $30^{\circ} \mathrm{C}$ for $10 \mathrm{~d}$. The suspension was added to seed medium which contained $5.0 \mathrm{~g}$ of starch, $5.0 \mathrm{~g}$ of glucose, $4.0 \mathrm{~g}$ of yeast extract, $5.0 \mathrm{~g}$ of $\mathrm{NaCl}, 2.0 \mathrm{~g}$ of $\left(\mathrm{NH}_{4}\right)_{2} \mathrm{SO}_{4}, 1.0 \mathrm{~g}$ of $\mathrm{MgSO}_{4} \cdot 7 \mathrm{H}_{2} \mathrm{O}$, and 1.0 of $\mathrm{g} \mathrm{K}_{2} \mathrm{HPO}_{4}$ in $1.0 \mathrm{~L}$ distilled water at a rate of $10^{8}$ spores in $50 \mathrm{~mL}$ medium. Cultures were kept on a shaker at $120 \mathrm{rpm}$ at $30^{\circ} \mathrm{C}$ for $3 \mathrm{~d}$ and used as seed stocks.

2.7. Selection of Basal Medium. Three different media, ISP4 [18], modified ISP4 [6], and yeast extract malt extract glucose medium [1], were used for comparative studies to find the basal nutrient medium for further formulation of optimal medium. $5 \mathrm{~mL}$ of the seed culture was transferred into $50 \mathrm{~mL}$ of different sterile medium in $150 \mathrm{~mL}$ flask. The flasks were incubated at $30^{\circ} \mathrm{C}$ on a rotary shaker at $150 \mathrm{rpm}$ for $10 \mathrm{~d}$. After the incubation, the cell-free supernatant was recovered for the extraction of antibiotic compound and assayed in triplicates for antibiotic activity to select an appropriate basal medium.

2.8. Optimization of Culture Conditions. Culture conditions, including incubation time and temperature, $\mathrm{pH}$, and $\mathrm{NaCl}$ concentration, were optimized to obtain an enhanced production of antibiotic from Nonomuraea sp JAJ18. All the determinations were carried out in triplicates with basal medium, which contained selected carbon and nitrogen sources from the previous experiment.

For determination of optimum incubation period, an Erlenmeyer flask (2L) containing $400 \mathrm{~mL}$ of the basal medium was inoculated with seed culture and incubated at $30^{\circ} \mathrm{C}$ in shaking incubator at $120 \mathrm{rpm}$ for $16 \mathrm{~d}$. At $24 \mathrm{~h}$ interval, $25 \mathrm{~mL}$ of culture broth was collected and centrifuged at $10000 \mathrm{rpm}$ for $10 \mathrm{~min}$. The crude compound was extracted from the mycelia-free supernatant and tested for antibiotic activity against Bacillus subtilis. The mycelial pellets were dried at $60^{\circ} \mathrm{C}$ and weighed to determine the growth.

A $\mathrm{pH}$ suitable for JAJ18 was determined using series of $250 \mathrm{~mL}$ Erlenmeyer flasks containing $50 \mathrm{~mL}$ of the basal medium adjusted to $\mathrm{pH} 5.5,6,6.5,7,7.5,8,8.5$, and 9 . Each flask was inoculated with $2 \% \mathrm{~V} / \mathrm{V}$ of seed culture. All of them were incubated at $30^{\circ} \mathrm{C}$ for $10 \mathrm{~d}$ in shaking incubator at $120 \mathrm{rpm}$. Crude compound was extracted and 
TABLE 1: Low and high levels of each variable used in Plackett-Burman design.

\begin{tabular}{lccc}
\hline Variables & Medium components & + values $(\mathrm{g} / \mathrm{L})$ & - values $(\mathrm{g} / \mathrm{L})$ \\
\hline$X_{1}$ & Starch & 20 & 2.0 \\
$X_{2}$ & $\left(\mathrm{NH}_{4}\right)_{2} \mathrm{SO}_{4}$ & 4 & 0.4 \\
$X_{3}$ & Yeast extract & 8 & 0.8 \\
$X_{4}$ & $\mathrm{NaCl}$ & 10 & 1.0 \\
$X_{5}$ & $\mathrm{MgSO}_{4} \cdot 7 \mathrm{H}_{2} \mathrm{O}$ & 2 & 0.2 \\
$X_{6}$ & $\mathrm{~K}_{2} \mathrm{HPO}_{4}$ & 2 & 0.2 \\
$X_{7}$ & $\mathrm{CaCO}_{3}$ & 4 & 0.4 \\
$X_{8}$ & $\mathrm{FeSO}_{4} \cdot 7 \mathrm{H}_{2} \mathrm{O}$ & 0.004 & 0.0004 \\
\hline
\end{tabular}

TABLE 2: Plackett-Burman design and experimental response obtained for Nonomuraea sp. JAJ18.

\begin{tabular}{|c|c|c|c|c|c|c|c|c|c|}
\hline \multirow{2}{*}{ Trial } & \multicolumn{8}{|c|}{ Variables } & \multirow{2}{*}{$\begin{array}{c}\text { Antibiotic effect }(\mathrm{U} / \mathrm{mL}) \pm \mathrm{SEM} \\
\text { B. subtilis }\end{array}$} \\
\hline & $X_{1}$ & $X_{2}$ & $X_{3}$ & $X_{4}$ & $X_{5}$ & $X_{6}$ & $X_{7}$ & $X_{8}$ & \\
\hline 1 & + & - & + & - & - & - & + & + & $120.0 \pm 10.0$ \\
\hline 2 & + & + & - & + & - & - & - & + & $103.3 \pm 10.4$ \\
\hline 3 & - & + & + & - & + & - & - & - & $106.7 \pm 11.5$ \\
\hline 4 & + & - & + & + & - & + & - & - & $80.0 \pm 10.0$ \\
\hline 5 & + & + & - & + & + & - & + & - & $126.7 \pm 5.78$ \\
\hline 6 & + & + & + & - & + & + & - & + & $138.3 \pm 2.89$ \\
\hline 7 & - & + & + & + & - & + & + & - & $70.0 \pm 10.0$ \\
\hline 8 & - & - & + & + & + & - & + & + & $106.7 \pm 5.78$ \\
\hline 9 & - & - & - & + & + & + & - & + & $86.7 \pm 5.78$ \\
\hline 10 & + & - & - & - & + & + & + & - & $115.0 \pm 8.67$ \\
\hline 11 & - & + & - & - & - & + & + & + & $95.0 \pm 13.2$ \\
\hline 12 & - & - & - & - & - & - & - & - & $86.7 \pm 5.78$ \\
\hline
\end{tabular}

antibiotic activity was tested against $B$. subtilis to determine the optimum $\mathrm{pH}$ for antibiotic production.

Similarly, for determination of optimum temperature, the Erlenmeyer flasks containing $50 \mathrm{~mL}$ of basal medium were inoculated with Nonomuraea sp. JAJ18 and incubated at different temperatures such as $20,25,30,37,40,45$, and $50^{\circ} \mathrm{C}$ for $10 \mathrm{~d}$ in shaking incubator at $120 \mathrm{rpm}$. Crude compound was extracted and antibiotic activity was assayed against $B$. subtilis to determine the optimum temperature for growth and antibiotic production from JAJ18.

2.9. Selection of the Best Carbon and Nitrogen Sources Using OFAT Method. In order to select the ideal carbon and nitrogen sources for the production of antibiotic from JAJ18, one-factor-at-a-time (OFAT) method was used. Various carbon (starch, sucrose, maltose, lactose, glucose, fructose, and glycerol) and nitrogen (yeast extract, peptone, urea, and ammonium sulfate) sources were used individually instead of the corresponding carbon and nitrogen sources in the chosen basal medium while other components were kept constant at original concentration, and the antibiotic activity was determined after $10 \mathrm{~d}$ of incubation at $30^{\circ} \mathrm{C}$ in a rotary shaker with $120 \mathrm{rpm}$. Combination of each nitrogen source with $0.4 \%(\mathrm{w} / \mathrm{v})$ yeast extract (YE) was also attempted.

2.10. Screening for Essential Medium Components Using Plackett-Burman Design (PBD). PBD was used to screen the medium components for their influence on antibiotic compound production by Nonomuraea sp. JAJ18. Minitab 15.0 (Minitab Inc., Pennsylvania, USA) was used for the development of experimental design and subsequent analysis of the experimental response data. Using the PBD, 8 medium components (independent variables) were screened by representing them at two levels (Table 1), low (-) and high (+) in 12 trials (Table 2). The experiments were carried out in triplicate and the average antibiotic activity against Bacillus subtilis was recorded as the response. Those variables showing confidence level above $95 \%$ were considered to have a significant influence on antibiotic production.

2.11. Optimization of Selected Ingredients by RSM. To find out the optimum levels of the selected media constituents, starch, $\mathrm{NaCl}$, and $\mathrm{MgSO}_{4} \cdot 7 \mathrm{H}_{2} \mathrm{O}$, for improving the antibiotic production by JAJ18, RSM was used with Box-Behnken design. The Design expert trial package (version 7) was used for the experimental design and the regression analysis of the data. The three medium components (independent variables) were taken as key input variables at three different levels, $(-)$, $(0)$, and (+) for low, intermediate, and high concentrations, respectively (Table 3 ). The concentrations of the other media components were fixed at zero level. Towards constructing a quadratic model, the experiment was performed in 17 trials (Table 4) with five replicates at the centre point and the values of responses were the mean of three replications. For predicting the optimal point, a second order model was fitted 
TABLE 3: Low, intermediate, and high levels of three independent variables used in RSM.

\begin{tabular}{lcccc}
\hline & Variables & & \multicolumn{3}{c}{ Range and level (g/L) } \\
& & + & 0 & - \\
\hline$A$ & Starch & 20 & 11 & 2 \\
$B$ & $\mathrm{NaCl}$ & 10 & 5.5 & 1 \\
$C$ & $\mathrm{MgSO}_{4} \cdot 7 \mathrm{H}_{2} \mathrm{O}$ & 2 & 0.55 & 0.2 \\
\hline
\end{tabular}

to correlate the relationship between independent variables and response. The behaviour of the system was explained by the following quadratic equation:

$$
Y=\beta_{0}+\Sigma \beta_{i} X_{i}+\Sigma \beta_{i j} X_{i} X_{j}+\Sigma \beta_{i i} X_{i}^{2},
$$

where $Y$ is the predicted response, $\beta_{0}$ is the intercept term, $\beta_{i}$ is the linear coefficient, $\beta_{i j}$ is the quadratic coefficient, and $\beta_{i i}$ is the interaction coefficient, with $X_{i} X_{j}$ representing the independent variables.

The statistical significance of the model was verified using the analysis of variance (ANOVA). Overall model significance was determined using Fisher's $F$-test and its associated probability $P(F)$. The quality of the polynomial model equation was tested statistically by coefficient of determination $\left(R^{2}\right)$ and adjusted $R^{2}$. The fitted polynomial equation was then expressed in the form of three-dimensional surface plots, to illustrate the relationship between the responses and the experimental levels of each independent variable. The Design Expert's numerical optimization method was employed to optimize the level of each variable for attaining maximum response.

2.12. Experimental Validation. The combination of different optimized variables, which gave the maximum response, was experimentally validated by culturing JAJ18 in optimized and unoptimized production medium in shake flasks. The cell-free culture broths were collected by centrifugation and extracted with equal volume of ethyl acetate; the top organic layer was concentrated and assayed for antibiotic activity.

\section{Results and Discussion}

3.1. Selection of the Optimal Nutrient Medium. Selection of basal medium is a prefatory and significant step for further formulation of an optimized medium to enhance antibiotic production by actinomycetes. In order to select a basal medium, strain JAJ18 was subjected to antibiotic production in three different media and tested for antibiotic activity. Modified ISP4 medium showed a maximum antibiotic activity $(120 \mathrm{U} / \mathrm{mL})$, followed by ISP4 medium $(90 \mathrm{U} / \mathrm{mL})$, and yeast extract malt extract glucose medium $(80 \mathrm{U} / \mathrm{mL})$. This is in agreement with previously reported employability of modified ISP4 for antibiotic production from actinomycetes of saltern origin [19]. Consequently, modified ISP4 medium was further used in a series of attempts to select suitable carbon and nitrogen sources and to improve antibiotic production by Nonomuraea sp. JAJ18 in batch cultures.

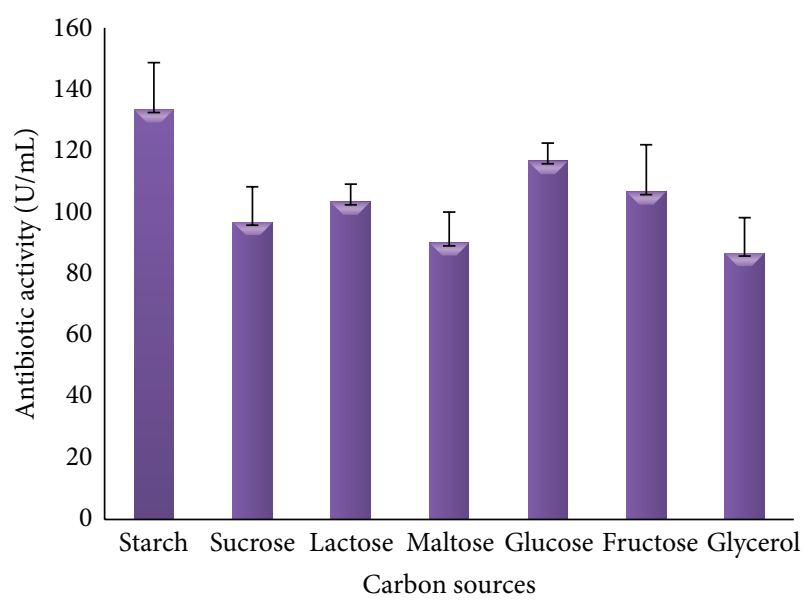

FIGURE 1: The effect of a range of simple and complex carbon sources on antibiotic activity of Nonomuraea sp. JAJ18.

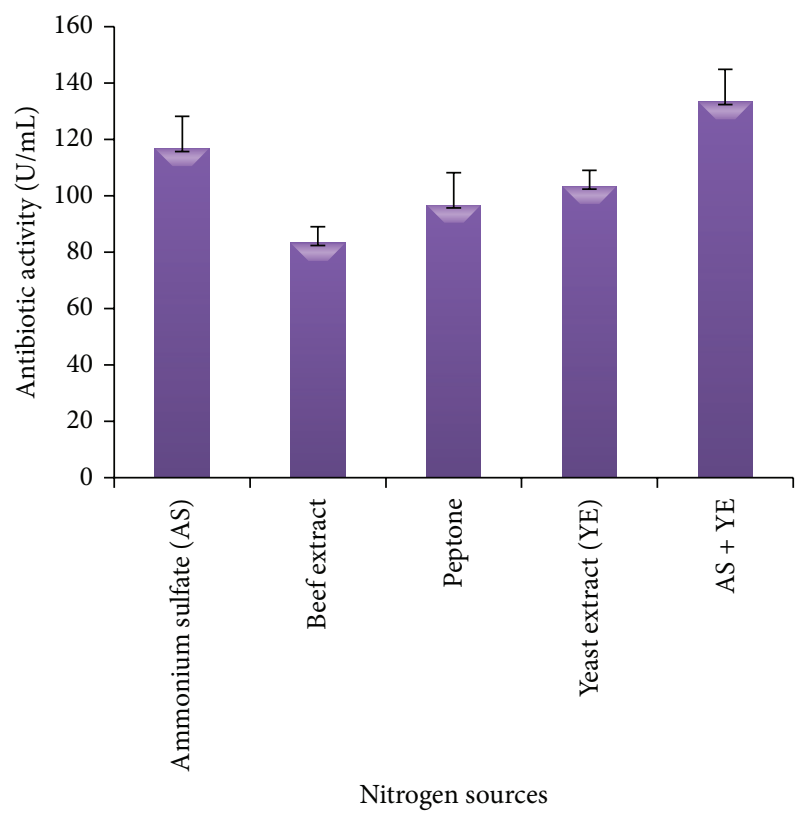

FIGURE 2: The effect of organic and inorganic nitrogen sources on antibiotic activity of Nonomuraea sp. JAJ18.

3.2. Selection of Carbon and Nitrogen Sources. Carbon and nitrogen sources are the vital constituents of culture media for better antibiotic production from actinomycetes [20,21]. Effects of various carbon and nitrogen sources on antibiotic activity of JAJ18 were studied with selected basal production medium and the results are presented in Figures 1 and 2. Among the various carbon sources studied, JAJ18 produced the maximum antibiotic activity in starch $(133.3 \mathrm{U} / \mathrm{mL})$ followed by glucose $(116.6 \mathrm{U} / \mathrm{mL})$. The antibiotic activity in other carbon sources, sucrose, lactose, maltose, fructose, and glycerol ranged from 96.6 to $106.6 \mathrm{U} / \mathrm{mL}$.

Likewise, among the various nitrogen sources, higher antibiotic production was favoured with ammonium sulfate $(116.6 \mathrm{U} / \mathrm{mL})$ followed by yeast extract $(103.3 \mathrm{U} / \mathrm{mL})$. 
TABLE 4: Box-Behnken design matrix used in RSM studies along with the experimental and predicted response (antibiotic activity).

\begin{tabular}{|c|c|c|c|c|c|}
\hline \multirow{2}{*}{ Runs } & \multicolumn{3}{|c|}{ Variables/coded values } & \multicolumn{2}{|c|}{ Antibiotic activity $(\mathrm{U} / \mathrm{mL}) \pm \mathrm{SEM}$} \\
\hline & Starch & $\mathrm{NaCl}$ & $\mathrm{MgSO}_{4} \cdot 7 \mathrm{H}_{2} \mathrm{O}$ & Experimental & Predicted \\
\hline 1 & - & - & 0 & $123.3 \pm 5.78$ & 122.94 \\
\hline 2 & + & - & 0 & $146.6 \pm 7.63$ & 145.81 \\
\hline 3 & - & + & 0 & $121.6 \pm 12.6$ & 122.39 \\
\hline 4 & + & + & 0 & $106.6 \pm 16.1$ & 106.96 \\
\hline 5 & - & 0 & - & $123.3 \pm 15.2$ & 123.13 \\
\hline 6 & + & 0 & - & $131.6 \pm 17.5$ & 131.85 \\
\hline 7 & - & 0 & + & $145.0 \pm 15.0$ & 144.75 \\
\hline 8 & + & 0 & + & $143.3 \pm 15.3$ & 143.48 \\
\hline 9 & 0 & - & - & $135.3 \pm 15.0$ & 135.84 \\
\hline 10 & 0 & + & - & $113.5 \pm 15.3$ & 112.89 \\
\hline 11 & 0 & - & + & $148.6 \pm 15.2$ & 149.21 \\
\hline 12 & 0 & + & + & $133.3 \pm 5.78$ & 132.76 \\
\hline 13 & 0 & 0 & 0 & $123.3 \pm 15.3$ & 122.62 \\
\hline 14 & 0 & 0 & 0 & $123.3 \pm 15.3$ & 122.62 \\
\hline 15 & 0 & 0 & 0 & $121.6 \pm 17.6$ & 122.62 \\
\hline 16 & 0 & 0 & 0 & $123.3 \pm 23.1$ & 122.62 \\
\hline 17 & 0 & 0 & 0 & $121.6 \pm 12.6$ & 122.62 \\
\hline
\end{tabular}

Lower antibiotic activities were observed with beef extract $(83.3 \mathrm{U} / \mathrm{mL})$ and peptone $(96.6 \mathrm{U} / \mathrm{mL})$. However, maximum antibiotic activity $(133.3 \mathrm{U} / \mathrm{mL})$ was observed with ammonium sulfate when supplemented with yeast extract. Thus, starch and combination of ammonium sulfate and yeast extract were chosen as the source of carbon and nitrogen for further experiments, respectively.

Starch, $\left(\mathrm{NH}_{4}\right)_{2} \mathrm{SO}_{4}$, and yeast extract were used in production media as the major nutrients for the production of cyclic peptide antibiotics in Nonomuraea species [22]. Besides that, there are no other reports on selection of carbon and nitrogen sources for antibiotic production by Nonomuraea. However, starch has been reported to be the major carbon source for antibiotic production by other actinomycetes, for instance, gentamicin production by Micromonospora echinospora [23]. Regarding nitrogen source, ammonium sulfate has widely been reported as a best nitrogen source for biomass as well as antibiotic production in many actinomycetes. Cimburkova et al. [24] reported improved production of avermectins from Streptomyces avermitilis using ammonium sulfate as the sole nitrogen source. Lee et al. [25] reported that ammonium sulfate is the best with respect to formation of antibiotic rapamycin and mycelial growth in Streptomyces hygroscopicus. Amending the production media with yeast extract has been widely reported to have significant influence on secondary metabolite production. In the present study, yeast extract was found to favour antibiotic production in JAJ18 which is in good agreement with a report which asserts that yeast extract was able to support relatively high antibiotic production in actinomycete, Streptomyces sp. IMV$70[26]$.

3.3. Selection of Culture Conditions. Optimization of culture conditions is necessary to improve antibiotic production [27].

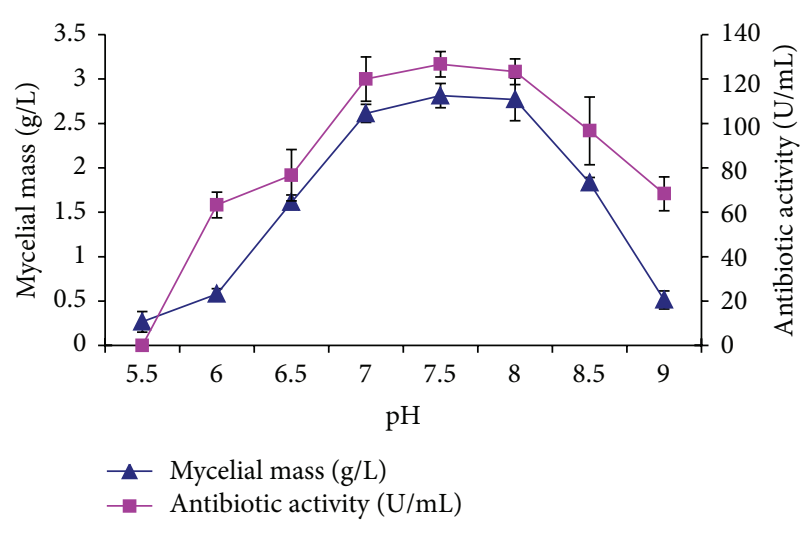

FIGURE 3: The effect of different initial $\mathrm{pH}$ on growth and antibiotic activity of Nonomuraea sp. JAJ18.

Optimum $\mathrm{pH}$, temperature, and incubation time for antibiotic production by Nonomuraea sp. JAJ18 were determined in series of preliminary experiments with basal production medium and are presented in Figures 3 and 4. Good growth as well as antibiotic activity was observed between $\mathrm{pH} 7$ and 8 with highest activity at $\mathrm{pH}$ 7.5. Likewise, the optimum temperature for growth and antibiotic production was found to be $32^{\circ} \mathrm{C}$. These results accord with a fact, that extreme $\mathrm{pH}$ and temperature are unfavourable for antibiotic production [28].

In order to select an appropriate incubation period to ensure maximum antibiotic recovery, antibiotic activity was studied at fixed time intervals by growing the antibiotic producing Nonomuraea sp. JAJ18 in shake flask for $15 \mathrm{~d}$ (Figure 4). The antibiotic activity was recorded after $4 \mathrm{~d}$ of growth and it attained the maximum in $11 \mathrm{~d}$, after the exponential growth phase. Thereafter, the mycelial biomass 
TABLE 5: Statistical analysis of effects of medium constituents on antibiotic activity as per PBD.

\begin{tabular}{lcccccc}
\hline Variables & Medium components & Effect & Standard error & $t$-value & $P$ value & Confidence level $(\%)$ \\
\hline$X_{1}$ & Starch & 21.91 & 1.863 & 5.88 & 0.010 & 99.0 \\
$X_{2}$ & $\left(\mathrm{NH}_{4}\right)_{2} \mathrm{SO}_{4}$ & 7.48 & 1.863 & 2.01 & 0.138 & 86.2 \\
$X_{3}$ & Yeast extract & 1.38 & 1.863 & 0.37 & 0.735 & 26.5 \\
$X_{4}$ & $\mathrm{NaCl}$ & -14.71 & 1.863 & -3.95 & 0.029 & 97.1 \\
$X_{5}$ & $\mathrm{MgSO}_{4} \cdot 7 \mathrm{H}_{2} \mathrm{O}$ & 20.85 & 1.863 & 5.60 & 0.011 & 9.062 \\
$X_{6}$ & $\mathrm{~K}_{2} \mathrm{HPO}_{4}$ & -10.85 & 1.863 & -2.91 & 0.251 & 93.8 \\
$X_{7}$ & $\mathrm{CaCO}_{3}$ & 5.28 & 1.863 & 1.42 & 0.062 & 74.9 \\
$X_{8}$ & $\mathrm{FeSO}_{4} \cdot 7 \mathrm{H}_{2} \mathrm{O}$ & 10.81 & 1.863 & 2.90 & 0.9 & 93.8 \\
\hline
\end{tabular}

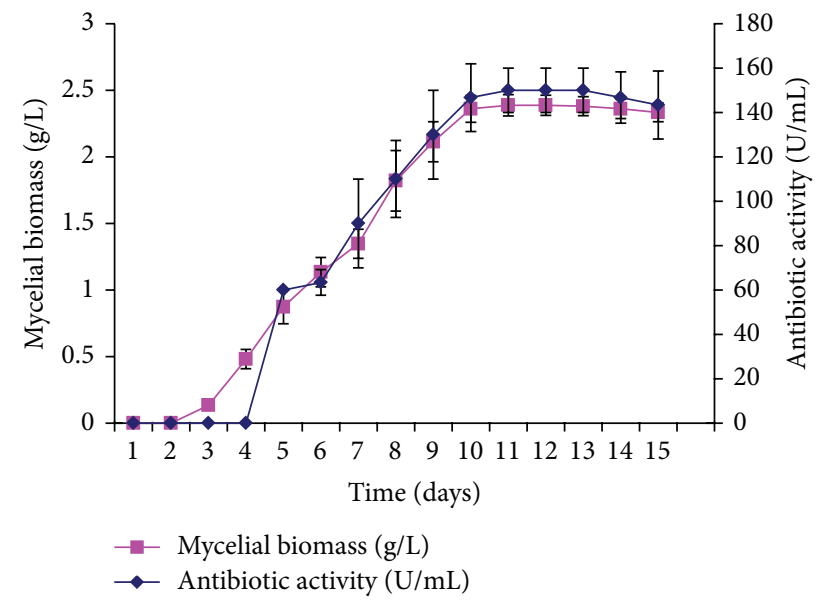

Figure 4: Effect of incubation period on growth and antibiotic activity of Nonomuraea sp. JAJ18.

and antibiotic activity remained constant for 3 days and then started to decline slightly. It was observed that degree of production of antibiotic was parallel with the biomass in production medium. The incubation time $(11 \mathrm{~d})$ required by this rare actinomycete Nonomuraea sp. JAJ18 to exert maximum antibiotic activity is higher than that reported for Streptomyces [29-31].

\subsection{Screening for Essential Medium Components Using PBD.}

The Plackett-Burman design has proven to be a valuable tool in screening and optimizing of media components and culture conditions in various bioprocesses including antibiotic production [14, 32, 33]. The Plackett-Burman experimental design was adopted with 12 trials to determine the medium components which significantly influence the antibiotic production by Nonomuraea sp. JAJ18. The effect, standard error, $t$-value, $P$ value, and confidence level of each component are shown in Table 5. The starch $\left(X_{1}\right)$, $\mathrm{NaCl}\left(X_{4}\right)$, and $\mathrm{MgSO}_{4} \cdot 7 \mathrm{H}_{2} \mathrm{O}\left(X_{5}\right)$ had significant effect on antibiotic production which was apparent from their confidence levels above $95 \%$ in comparison to other variables. It was further confirmed from Pareto chart (Figure 5), in which the maximal effect is presented in the upper portion and then progresses down to the minimal effect. Therefore the medium components, starch, $\mathrm{NaCl}$ and $\mathrm{MgSO}_{4} \cdot 7 \mathrm{H}_{2} \mathrm{O}$,

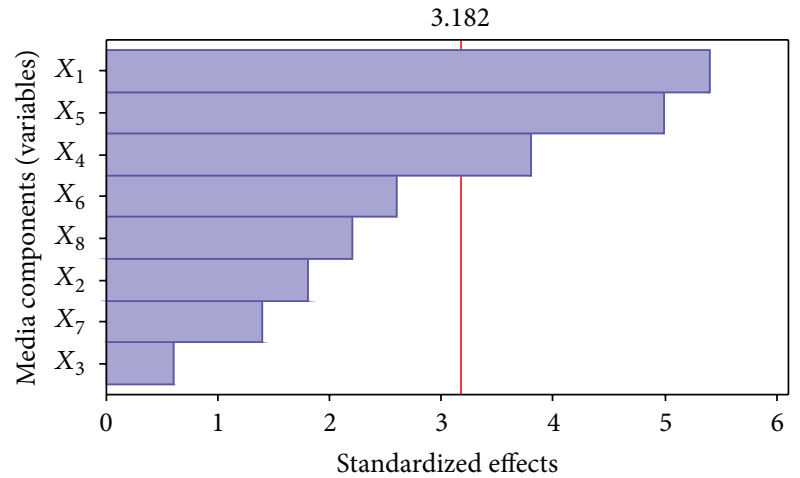

FIgURE 5: Pareto chart shows the effects of variables on antibiotic activity of Nonomuraea sp. JAJ18 investigated in the PlackettBurman design.

were considered as significant components for further optimization using response surface methodology to ensure the optimum antibiotic production by JAJ18.

3.5. Optimization of Selected Ingredients Using RSM. Several researchers working on antibiotics discovery programs have applied PBD and RSM as statistical tools to recognize, manipulate, and optimize influencing medium constituents and recorded the increased antibiotic production [12, 34]. RSM with Box-Behnken design was applied to determine the optimal levels of the three selected variables (starch, $\mathrm{NaCl}$, and $\mathrm{MgSO}_{4} \cdot 7 \mathrm{H}_{2} \mathrm{O}$ ) that affected the production of antibiotic by JAJ18. A batch of experiments was conducted according to Box-Behnken design and the results were summarised along with the predicted values (Table 4) to determine the effect of independent factors on the response. The experimental results were evaluated and the following model equation was obtained for the three-factor system. Consider

$$
\begin{aligned}
& Y(\text { Antibiotic activity }) \\
&= 122.62+1.86 A-9.85 B+8.31 C \\
&-9.57 A B-2.50 A C+1.63 B C+2.51 A^{2} \\
&-0.61 B^{2}+10.67 C^{2},
\end{aligned}
$$


TABLE 6: ANOVA of quadratic polynomial model and significance test.

\begin{tabular}{lccc}
\hline Source & Sum of squares & df & Mean square \\
\hline Model & 2277.93 & 9 & 253.10 \\
Residual & 6.49 & 7 & 0.93 \\
Lack of fit & 3.02 & 3 & 1.01 \\
Pure error & 3.47 & 4 & 0.87 \\
\hline Total & 2284.42 & 16 & \\
\hline$R^{2}=0.9972$, Adj $R^{2}=0.9935$. & & \\
\end{tabular}

(a)

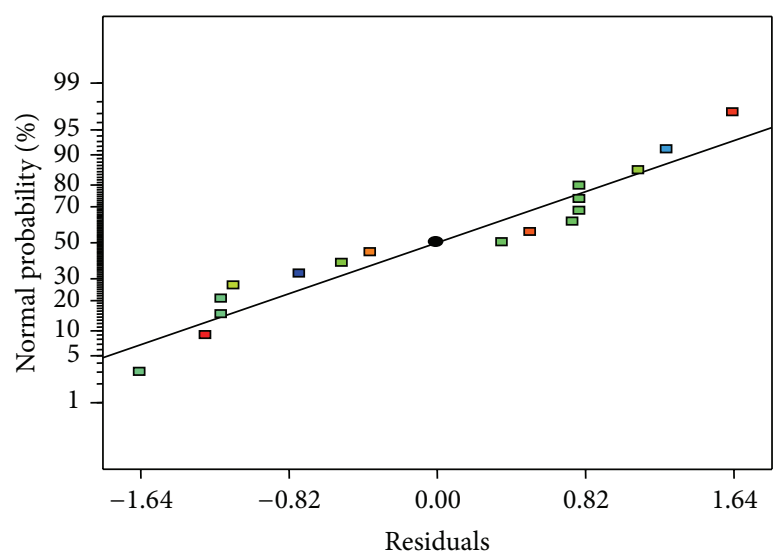

(b)

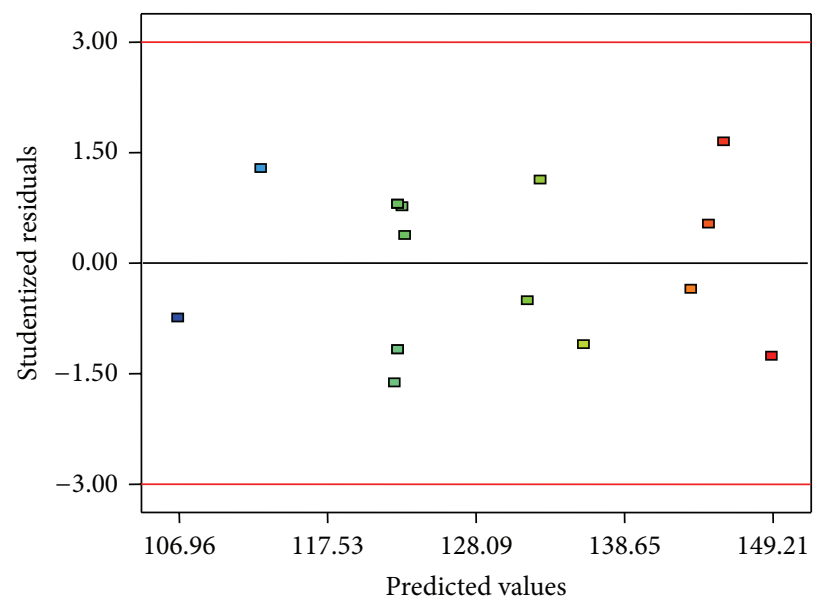

(c)

FIGURE 6: Residual diagnostic plots of quadratic model. Observed versus predicted reponse plot (a): normal probability plot of the studentized residuals (b); internally studentized residuals versus predicted response plot (c).

where $Y$ was the predicted response (antibiotic activity) and $A, B$, and $C$ were the coded values of starch, $\mathrm{KBr}$ and $\mathrm{CaCO}_{3}$, respectively.

The analysis of variance (ANOVA) was performed to statistically inspect the quadratic regression model and the results are summarised in Table 6 . The results showed that the model was highly significant, as it was evident from Fisher's $F$-test with low $P$ value $(<0.0001)$. Significance of model was further supported by statistically insignificant lack of fit, as was evident from the lower calculated $F$-value (1.16). Accuracy of the model can be checked by the determination of coefficient of $R^{2}$. The closer the value of $R^{2}$ to 1 , the stronger the model to predict the response [34]. The model $R^{2}$ of 0.9972 implied that model equation could explain $99.72 \%$ of the total variation in the response. The observed $R^{2}$ value was comparable with the earlier reports $[12,35]$.

In order to judge the model adequacy and clarify the signs of any problems in the experimental data, a series of diagnostic plots can be used [12]. Plot of observed versus predicted response is shown in Figure 6(a). In this case, predicted values were in agreement with observed ones in the range of the operating variables, which indicated goodness 


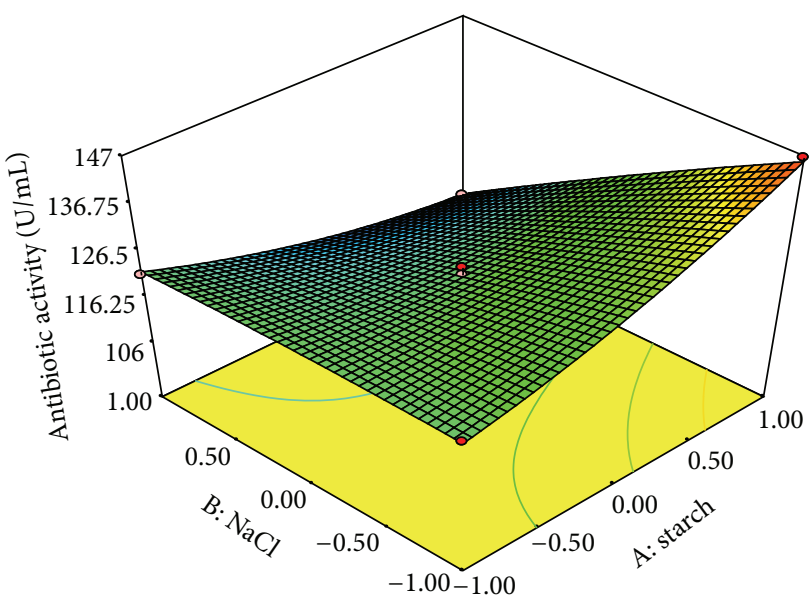

(a)

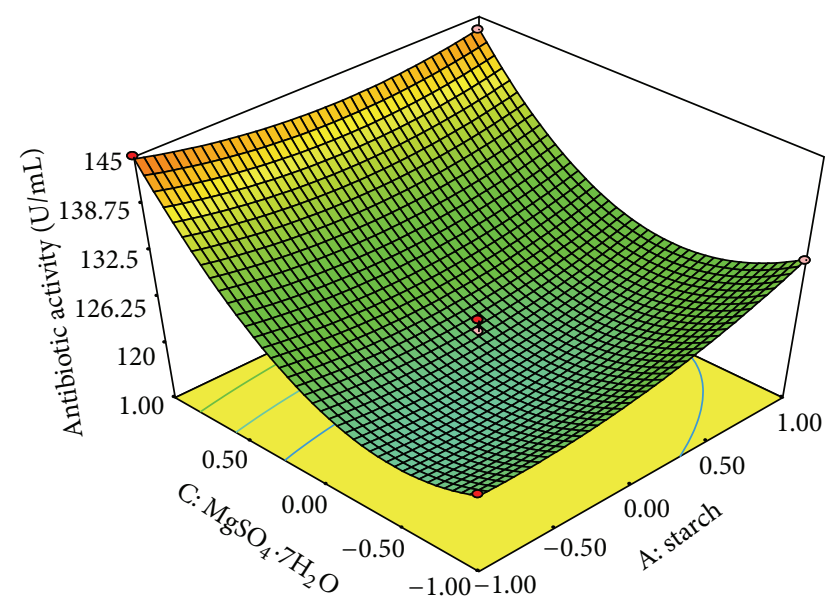

(b)

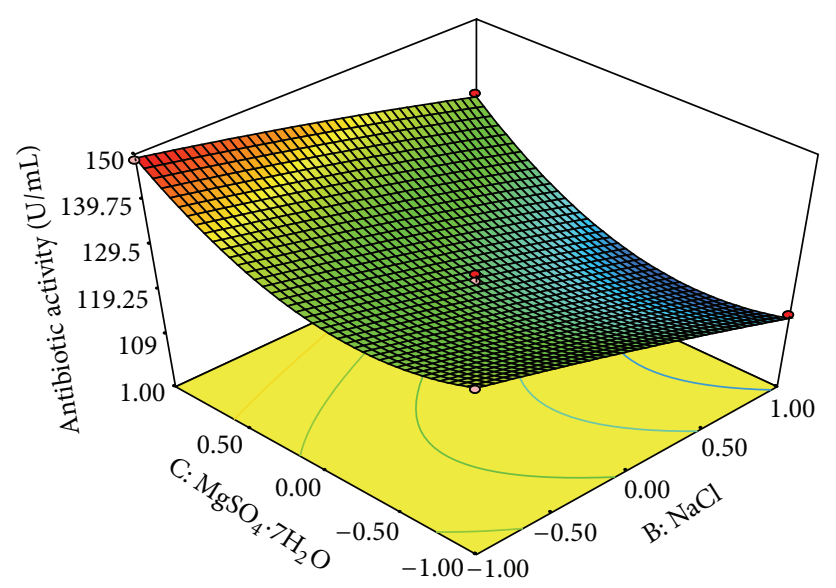

(c)

FIGURE 7: Response surface plots showing individual and interactive effects of variables on antibiotic activity by Nonomuraea sp. JAJ18. Effects of strach and $\mathrm{NaCl}$ on antibiotic activity: (a) effects of starch and $\mathrm{MgSO}_{4} \cdot 7 \mathrm{H}_{2} \mathrm{O}$ on antibiotic activity; (b) effects of $\mathrm{NaCl}_{\text {and }} \mathrm{MgSO}_{4} \cdot 7 \mathrm{H}_{2} \mathrm{O}$ on antibiotic activity.

and correctness of the model. The normal probability plot of the studentized residuals was drawn to check for normality of residuals (Figure 6(b)). A linear pattern observed in this plot indicated that there was no sign of any problem in the experimental data. A plot of studentized residuals versus predicted values was drawn to check for constant error (Figure 6(c)). Residuals displayed randomness in scattering and suggested that the variance of the original observation was constant.

Three-dimensional (3D) plots assisted in understanding the main as well as the interaction effects of three factors, starch, $\mathrm{NaCl}$ and $\mathrm{MgSO}_{4} \cdot 7 \mathrm{H}_{2} \mathrm{O}$. The 3D response surface plots were drawn to illustrate the pairwise combination of the three variables, while the rest was held at middle level (Figure 7). It is obvious from the plots that higher concentration of starch and $\mathrm{MgSO}_{4} \cdot 7 \mathrm{H}_{2} \mathrm{O}$ and lower concentration of $\mathrm{NaCl}$ favour higher antibiotic production. With the increase in concentration of starch from 0.02 to $2 \mathrm{~g} / \mathrm{L}$ (coded values, -1 to +1 ), the antibiotic activity gradually increased to a maximum at low concentration of $\mathrm{NaCl}$ (Figure 7(a)). However, the antibiotic activity was significantly suppressed when the concentration of $\mathrm{NaCl}$ was increased to higher levels in production medium. When the concentration of $\mathrm{MgSO}_{4} \cdot 7 \mathrm{H}_{2} \mathrm{O}$ was near to middle level (coded values, -1 to 0.0 ), the antibiotic activity increased with increasing concentration of starch from 2 to $20 \mathrm{~g} / \mathrm{L}$ (coded values, -1 to +1). Further increase of $\mathrm{MgSO}_{4} \cdot 7 \mathrm{H}_{2} \mathrm{O}$ to its high level (coded values, 0.0 to +1 ) resulted in significant increase in antibiotic production even at lower concentrations of starch (Figure 7(b)). With the increase of $\mathrm{NaCl}$ concentration from 1 to $10 \mathrm{~g} / \mathrm{L}$ (coded values, -1.0 to +1.0 ), the antibiotic activity gradually decreased at lower concentration of $\mathrm{MgSO}_{4} \cdot 7 \mathrm{H}_{2} \mathrm{O}$ (coded values, -1.0 to -0.5 ); however, intensity of decrease was significantly reduced with increasing concentration of $\mathrm{MgSO}_{4} \cdot 7 \mathrm{H}_{2} \mathrm{O}$ (Figure $7(\mathrm{c})$ ).

Using numerical optimization method, predicted maximum antibiotic activity was $154.49 \mathrm{U} / \mathrm{mL}$ (Figure 8), when the optimal values of test factors in the coded units were 


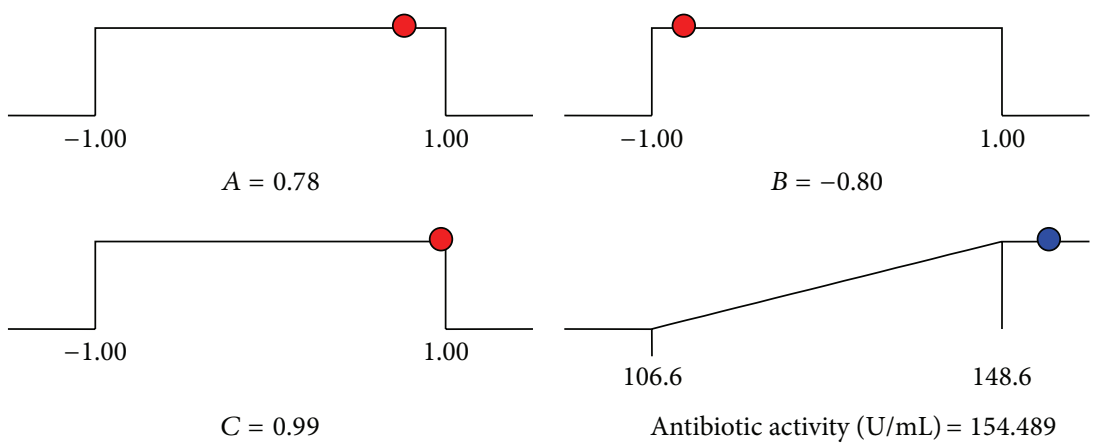

FIGURE 8: Summary of criteria set for optimization run. Ramps shows the predicted levels of variables within the range of given concentration (coded values) and predicted possible antibiotic activity of JAJ18.

TABLE 7: Experimental validation of the combined effect of variables under optimized and unoptimized conditions on the antibiotic activity of Nonomuraea sp. JAJ18.

\begin{tabular}{|c|c|c|c|c|c|}
\hline \multirow{2}{*}{ Variables } & \multicolumn{2}{|c|}{ Level $(\mathrm{g} / \mathrm{L})$} & \multicolumn{3}{|c|}{ Antibiotic activity $(\mathrm{U} / \mathrm{mL})$} \\
\hline & Unoptimized & Optimized & Unoptimized & Optimized (predicted) & Optimized (experimental) \\
\hline Starch & 10 & 15.6 & & & \\
\hline $\mathrm{NaCl}$ & 5 & 0.8 & 140.0 & 154.49 & 161.7 \\
\hline $\mathrm{MgSO}_{4} \cdot 7 \mathrm{H}_{2} \mathrm{O}$ & 1 & 1.98 & & & \\
\hline
\end{tabular}

starch $=0.78, \mathrm{NaCl}=-0.80$ and $\mathrm{MgSO}_{4} \cdot 7 \mathrm{H}_{2} \mathrm{O}=0.99$, which were $15.6 \mathrm{~g} / \mathrm{L}$ starch, $0.8 \mathrm{~g} / \mathrm{L} \mathrm{NaCl}$ and $1.98 \mathrm{~g} / \mathrm{L} \mathrm{MgSO}_{4} \cdot 7 \mathrm{H}_{2} \mathrm{O}$, respectively.

3.6. Experimental Validation. In order to validate the statistical results, a verification experiment was performed in triplicate with a predicted optimal medium in shake flasks (Table 7). The antibiotic activity unit obtained experimentally was $161.7 \mathrm{U} / \mathrm{mL}$, which is in reasonable agreement with the maximum predicted value $(154.49 \mathrm{U} / \mathrm{mL})$. This result proves the aptness of the model for predicting the production of antibiotic by Nonomuraea sp. JAJ18. The final optimized medium contained $15.6 \mathrm{~g}$ of starch, $2.0 \mathrm{~g}$ of $\left(\mathrm{NH}_{4}\right)_{2} \mathrm{SO}_{4}, 4.0 \mathrm{~g}$ of yeast extract, $0.8 \mathrm{~g}$ of $\mathrm{NaCl}, 1.98 \mathrm{~g}$ of $\mathrm{MgSO}_{4} \cdot 7 \mathrm{H}_{2} \mathrm{O}, 1 \mathrm{~g}$ of $\mathrm{K}_{2} \mathrm{HPO}_{4}, 2 \mathrm{~g}$ of $\mathrm{CaCO}_{3}$, and $0.004 \mathrm{~g}$ of $\mathrm{FeSO}_{4} \cdot 7 \mathrm{H}_{2} \mathrm{O}$ in $1 \mathrm{~L}$ of distilled water.

\section{Conclusion}

To the best of our knowledge, this is the first report on improved antibiotic activity of Nonomuraea sp. by medium optimization using a combination of nonstatistical and statistical methods. The study focused primarily on improved production of antibiotic by Nonomuraea sp. JAJ18 as a function of various carbon and nitrogen sources and levels of ingredients in production medium as well as culture conditions. The results support the use of nonstatistical onefactor-at-a-time method for recruitment of suitable carbon and nitrogen sources. Further, PBD and RSM were found to be very effective in determining and optimizing the medium components in manageable number of experimental trials with overall $15.5 \%$ increase in antibiotic activity. The optimum culture medium and the culture conditions defined from this study will be useful for further development of large scale fermentation process for the efficient production of antibiotic from this Nonomuraea sp. JAJ18.

\section{Conflict of Interests}

The authors declare that they have no conflict of interests.

\section{Acknowledgment}

The authors acknowledge the financial support offered by the Council of Scientific and Industrial Research-Human Resource Development Group (CSIR-HRDG), India.

\section{References}

[1] B. Badji, A. Mostefaoui, N. Sabaou et al., "Isolation and partial characterization of antimicrobial compounds from a new strain Nonomuraea sp. NM94," Journal of Industrial Microbiology and Biotechnology, vol. 34, no. 6, pp. 403-412, 2007.

[2] A. Lazzarini, L. Cavaletti, G. Toppo, and F. Marinelli, "Rare genera of actinomycetes as potential producers of new antibiotics," Antonie van Leeuwenhoek, vol. 78, no. 3-4, pp. 399-405, 2001.

[3] K. Tiwari and R. K. Gupta, "Rare actinomycetes: a potential storehouse for novel antibiotics," Critical Reviews in Biotechnology, vol. 32, no. 2, pp. 108-132, 2012.

[4] P. A. Jose and S. R. D. Jebakumar, "Non-streptomycete actinomycetes nourish the current microbial antibiotic drug discovery," Frontiers in Microbiology, vol. 4, no. 240, 2013.

[5] K. Tiwari and R. K. Gupta, "Diversity and isolation of rare actinomycetes: an overview," Critical Reviews in Microbiology, vol. 39, no. 3, pp. 256-294, 2013. 
[6] P. A. Jose and S. R. D. Jebakumar, "Diverse actinomycetes from Indian coastal solar salterns-a resource for antimicrobial screening," Journal of Pure and Applied Microbiology, vol. 7, no. 4, pp. 2569-2575, 2013.

[7] R. L. Greasham, "Media for microbial fermentations," in Biotechnology: Volume 3: Bioprocessing, H. J. Rehm, G. Read, A. Puhler, and P. Stagler, Eds., pp. 128-139, Wiley-VCH, New York, NY, USA, 1983.

[8] N. Gunnarsson, P. Bruheim, and J. Nielsen, "Production of the glycopeptide antibiotic A40926 by Nonomuraea sp. ATCC 39727: influence of medium composition in batch fermentation," Journal of Industrial Microbiology and Biotechnology, vol. 30, no. 3, pp. 150-156, 2003.

[9] M. A. Hassan, M. Y. El-Naggar, and W. Y. Said, "Physiological factors affecting the production of an antimicrobial substance by Streptomyces violatus in batch cultures," Egyptian Journal of Biology, vol. 3, pp. 1-10, 2001.

[10] Y. H. Wang, J.-T. Feng, Q. Zhang, and X. Zhang, "Optimization of fermentation condition for antibiotic production by Xenorhabdus nematophila with response surface methodology," Journal of Applied Microbiology, vol. 104, no. 3, pp. 735-744, 2008.

[11] J. Feng, W. Zhang, L. Han, and X. Zhang, "Statistical optimization of medium components to improve the antibiotic activity of Streptomyces sp. 19G-317," African Journal of Agricultural Research, vol. 6, no. 19, pp. 4424-4431, 2011.

[12] Y. Wang, X. Fang, F. An, G. Wang, and X. Zhang, "Improvement of antibiotic activity of Xenorhabdus bovienii by medium optimization using response surface methodology," Microbial Cell Factories, vol. 10, article 98, 2011.

[13] C. Xu and J. Yun, "Optimization of submerged-culture conditions for mycelial growth and exo-biopolymer production by Auricularia polytricha (wood ears fungus) using the methods of uniform design and regression analysis," Biotechnology and Applied Biochemistry, vol. 38, no. 2, pp. 193-199, 2003.

[14] N. Singh and V. Rai, "Improved antimicrobial compound production by a new isolate Streptomyces hygroscopicus MTCC 4003 using Plackett-Burman design and response surface methodology," Bioinformation, vol. 8, pp. 1021-1025, 2012.

[15] K. Adinarayana, P. Ellaiah, B. Srinivasulu, R. Bhavani Devi, and G. Adinarayana, "Response surface methodological approach to optimize the nutritional parameters for neomycin production by Streptomyces marinensis under solid-state fermentation," Process Biochemistry, vol. 38, no. 11, pp. 1565-1572, 2003.

[16] P. A. Jose and S. R. D. Jebakumar, "Phylogenetic diversity of actinomycetes cultured from coastal multipond solar saltern in Tuticorin, India," Aquatic Biosystems, vol. 8, article 23, no. 1, 2012.

[17] P. W. Maxwell, G. Chen, J. M. Webster, and G. B. Dunphy, "Stability and activities of antibiotics produced during infection of the insect Galleria mellonella by two isolates of Xenorhabdus nematophilus," Applied and Environmental Microbiology, vol. 60, no. 2, pp. 715-721, 1994.

[18] E. Küster, "Outline of a comparative study of criteria used in characterization of the actinomycetes," Intetnational Bulletin of Bacteriological Nomenclature and Taxonomy, vol. 9, pp. 97-104, 1959.

[19] P. A. Jose and S. R. D. Jebakumar, "Phylogenetic appraisal of antagonistic, slow growing actinomycetes isolated from hypersaline inland solar salterns at Sambhar Salt Lake, India," Frontiers in Microbiology, vol. 4, article 190, 2013.
[20] G. Hobbs, C. M. Frazer, D. C. J. Gardner, F. Flett, and S. G. Oliver, "Pigmented antibiotic production by Streptomyces coelicolor A3(2): Kinetics and the influence of nutrients," Journal of General Microbiology, vol. 136, no. 11, pp. 2291-2296, 1990.

[21] A. Pandey, A. Shukla, and S. K. Majumdar, "Utilization of carbon and nitrogen sources by Streptomyces kanamyceticus M 27 for the production of an anti bacterial antibiotic," African Journal of Biotechnology, vol. 4, no. 9, pp. 909-910, 2005.

[22] S. Cho, J. K. Choi, S. Franzblau et al., "Cyclic peptide from Nonomuraea sp., process for the production thereof, and pharmaceutical composition for the prevention or treatment of mycobacteria related disease comprising the same," Patent WO2012144790 A1, 2012.

[23] M. Himabindu and A. Jetty, "Optimization of nutritional requirements for gentamicin production by Micromonospora echinospora," Indian Journal of Experimental Biology, vol. 44, no. 10, pp. 842-848, 2006.

[24] E. Cimburkova, J. Zima, J. Novak, and Z. Vanek, "Nitrogen regulation of avermectins biosynthesis in Streptomyces avermitilis in a chemically defined medium," Journal of Basic Microbiology, vol. 28, no. 8, pp. 491-499, 1988.

[25] M. S. Lee, I. Kojima, and A. L. Demain, "Effect of nitrogen source on biosynthesis of rapamycin by Streptomyces hygroscopicus," Journal of Industrial Microbiology and Biotechnology, vol. 19, no. 2, pp. 83-86, 1997.

[26] L. P. Trenozhnikova, A. K. Khasenova, A. S. Balgimbaeva et al., "Characterization of the antibiotic compound no. 70 produced by Streptomyces sp. IMV-70," The Scientific World Journal, vol. 2012, Article ID 594231, 8 pages, 2012.

[27] X.-L. Fang, L.-R. Han, X.-Q. Cao, M.-X. Zhu, X. Zhang, and Y.-H. Wang, "Statistical optimization of process variables for antibiotic activity of Xenorhabdus bovienii," PLoS ONE, vol. 7, no. 6, Article ID e38421, 2012.

[28] M. Rostamza, A. Noohi, and Y. Hamedi, "Enhancement in production of erythromycin by Saccharopolyspora erythraea by the use of suitable industrial seeding-media," $D A R U$, vol. 16, no. 1, pp. 13-17, 2008.

[29] A. K. Paul and A. K. Banerjee, "Determination of optimum conditions for antibiotic production by Streptomyces galbus," Folia Microbiologica, vol. 28, no. 5, pp. 397-405, 1983.

[30] H. M. Atta, B. M. Haroun, and M. A. Khalifa, "Physicochemical characteristics of vernamycin-A antibiotic biosynthesis by Streptomyces SP-AZ-SH-29," Journal of Saudi Chemical Society, vol. 15, no. 3, pp. 247-255, 2011.

[31] C. K. M. Tripathi, M. Khan, V. Praveen, S. Khan, and A. Srivastava, "Enhanced antibiotic production by Streptomyces sindenensis using artificial neural networks coupled with genetic algorithm and Nelder-Mead downhill simplex," Journal of Microbiology and Biotechnology, vol. 22, no. 7, pp. 939-946, 2012.

[32] V. Gohel, T. Chaudhary, P. Vyas, and H. S. Chhatpar, "Statistical screenings of medium components for the production of chitinase by the marine isolate Pantoea dispersa," Biochemical Engineering Journal, vol. 28, no. 1, pp. 50-56, 2006.

[33] Z. Guo, L. Shen, Z. Ji, and W. Wu, "Enhanced production of a novel cyclic hexapeptide antibiotic (NW-G01) by Streptomyces antibiotic 313 using response surface methodology," International Journal of Molecular Sciences, vol. 13, no. 4, pp. 5230-5241, 2012.

[34] H. Chen, M. Wu, Z. Chen, M. Wang, J. Lin, and L. Yang, "Enhancing production of a 24-membered ring macrolide compound by a marine bacterium using response surface 
methodology," Journal of Zhejiang University SCIENCE B, vol. 14, pp. 346-354, 2013.

[35] P. A. Jose, K. K. Sivakala, and S. R. D. Jebakumar, "Formulation and statistical optimization of culture medium for improved production of antimicrobial compound by Streptomyces sp. JAJ06," International Journal of Microbiology, vol. 2013, Article ID 526260, 9 pages, 2013. 

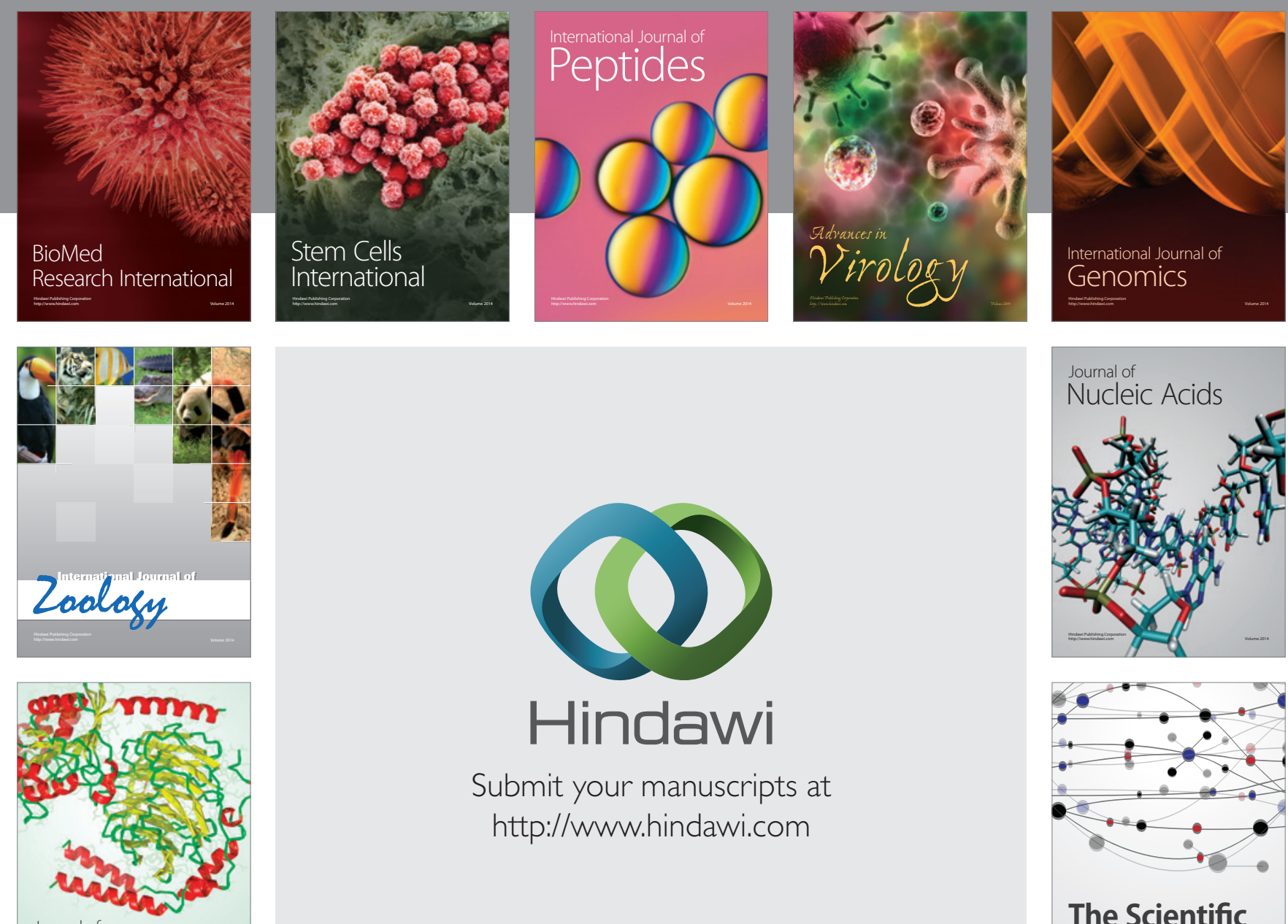

Submit your manuscripts at

http://www.hindawi.com

Journal of
Signal Transduction
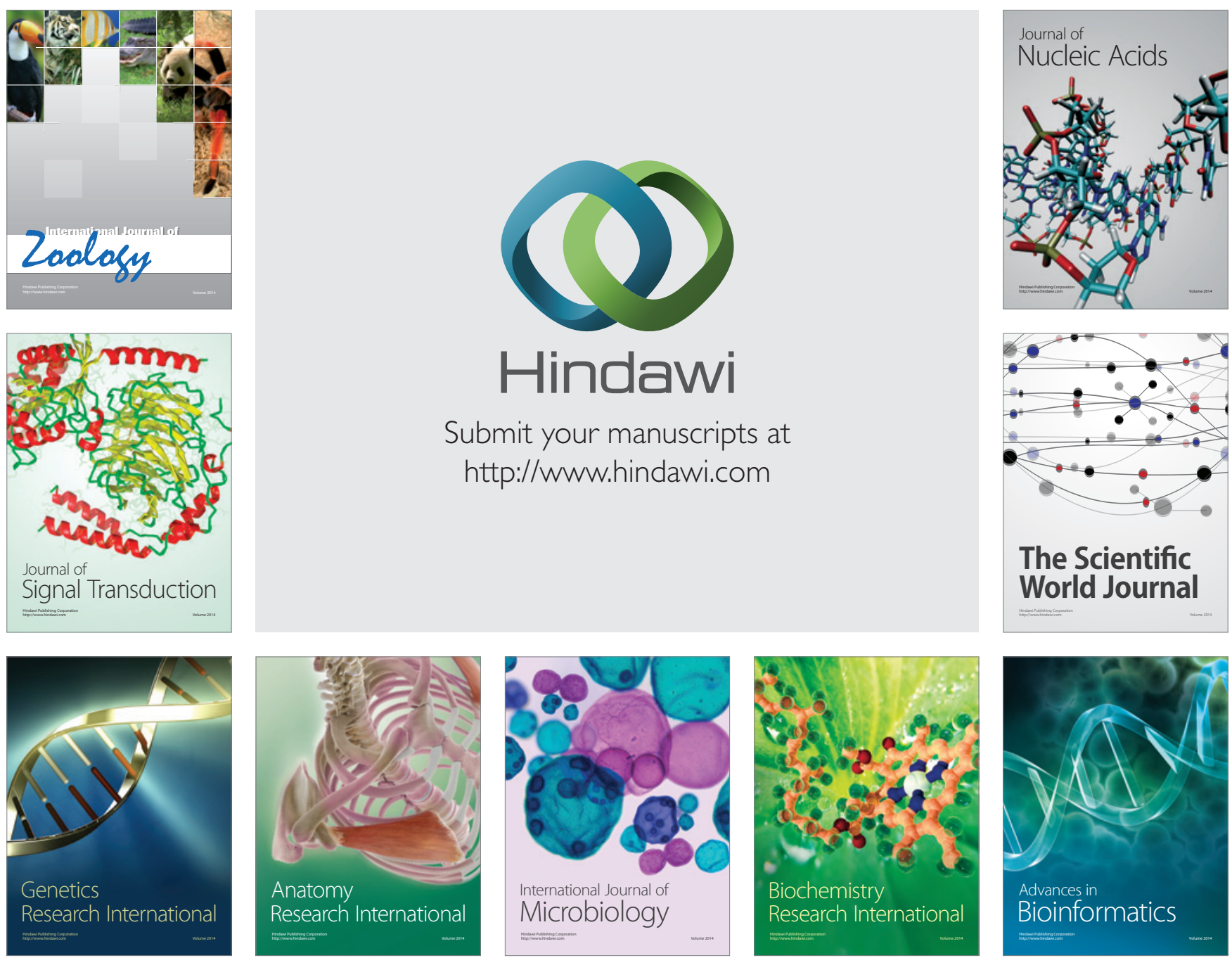

The Scientific World Journal
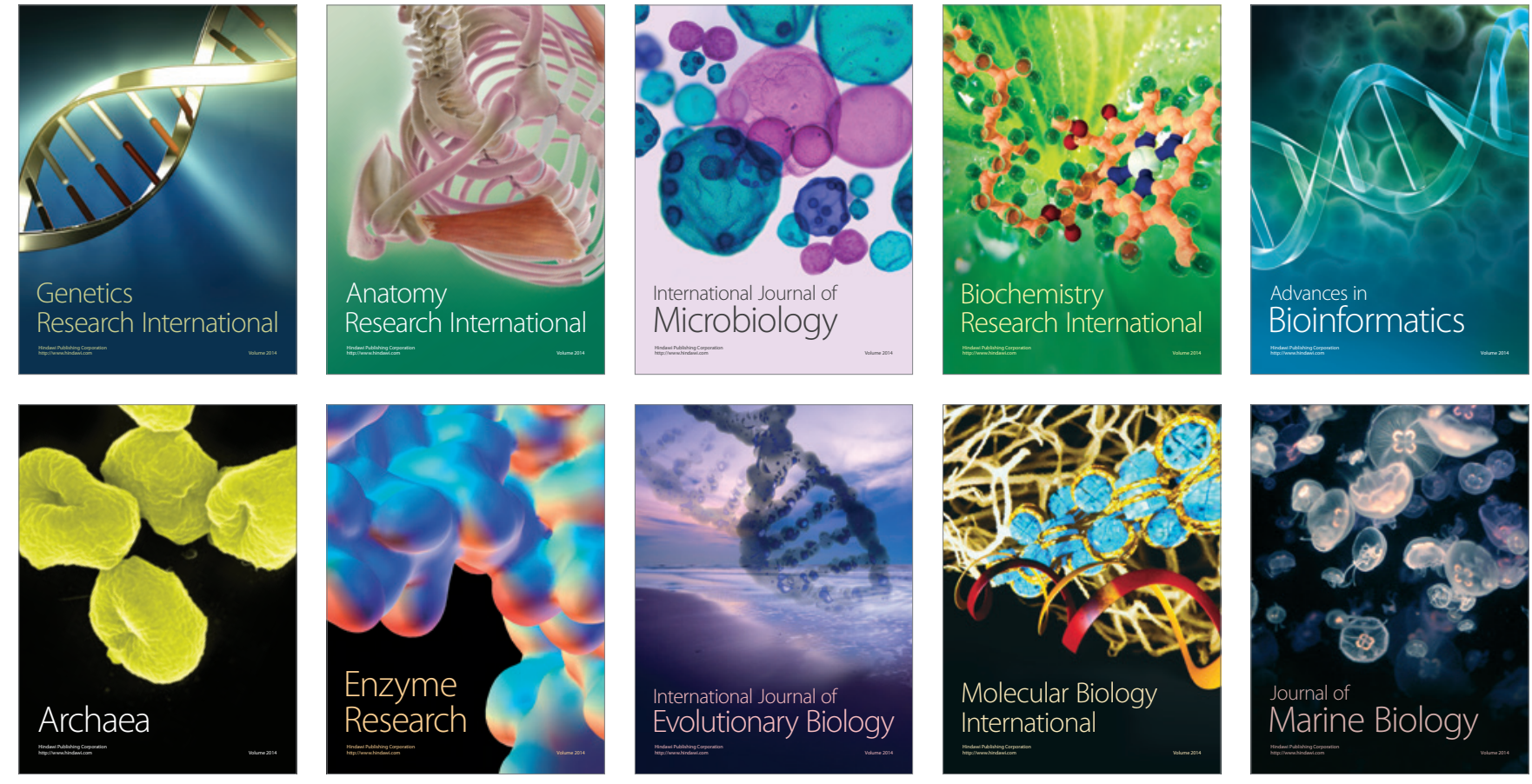